\title{
3rd International Workshop on Image Mining. Theory and Applications (IMTA-3-2010) ${ }^{1}$
}

\author{
May 20, 2010-Angers, France
}

DOI: $10.1134 / \mathrm{S} 1054661811010123$

In conjunction with the 3rd International Joint Conference on Computer Vision, Imaging and Computer Graphics Theory and Applications-VISIGRAPP 2010.

This special issue of the journal "Pattern Recognition and Image Analysis" contains the texts of papers presented (and submitted for publication in this issue) at the 3rd International Workshop on Image Mining held in May 2009 in Angers, France.

The workshop was organized in the framework of the 3rd International Joint Conference on Computer Vision, Imaging and Computer Graphics Theory and Applications (VISIGRAPP 2010) in cooperation with the Technical Committee no. 16 "Algebraic and Discrete Mathematical Techniques in Pattern Recognition and Image Analysis" of the International Association for Pattern Recognition and with the National Committee for Pattern Recognition and Image Analysis of the Russian Academy of Sciences.

Taking as a strategic goal the automated image mining it is necessary to provide image analysis professionals and final users with the following opportunities:

- automated design, test and adaptation of techniques and algorithms for image recognition, estimation and understanding;

- automated selection of techniques and algorithms for image recognition, estimation and understanding;

- automated testing of the raw data quality and suitability for solving the image recognition problem;

- standard technological schemes for image recognition, estimation, understanding and retrieval.

Automation of image-mining is possible by combining and applying of different mathematical techniques for image analysis, understanding and recognition, in particular of algebraic and discrete mathematics techniques.

Automation of image processing, analysis, estimating and understanding is one of the crucial points of theoretical computer science having decisive importance for applications, in particular, for diversification

\footnotetext{
${ }^{1}$ The article is published in the original.
}

of solvable problem types and for increasing the efficiency of problem solving.

The role of an image as an analysis and estimation object is determined by its specific and inalienable informational properties. Image is a mixture and a combination of initial (raw, "real") data and its representation means, of computational and physical nature and models of objects, events and processes to be represented via an image.

The specificity, complexity and difficulties of image analysis and estimation (IAE) problems stem from necessity to achieve some balance between such highly contradictory factors as goals and tasks of a problem solving, the nature of visual perception, ways and means of an image acquisition, formation, reproduction and rendering, and state of the art in the mathematical, computational and technological means allowable for the IAE.

We may consider that the main contradiction is related to the "pictorial nature" of an image and the "formal" (symbolic) foundations of IAE: it is well known that to take an advantage from data representation an image form is necessary to reduce the latter to a "non-image" form.

In IAE is used a wide spectrum of mathematical techniques from algebra, geometry, discrete mathematics, mathematical logics, probability theory, mathematical statistics, calculus, as well as the techniques of mathematical theory of pattern recognition, digital signal processing, and physics (in particular, optics).

The mathematical theory of image analysis is not finished and is passing through a developing stage. It is only recently came understanding of the fact that only intensive creating of comprehensive mathematical theory of image analysis and recognition (in addition to the mathematical theory of pattern recognition) could bring a real opportunity to solve efficiently application problems via extracting from images the information necessary for intellectual decision making.

The transition to practical, reliable and efficient automation of image-mining is directly dependent on introducing and developing of mathematical means for IAE.

The natural way to overcome the above mentioned contradiction between "pictorial nature" of an image and the "formal" (symbolic) foundations of IAE is to 
introduce pattern recognition oriented image models and necessary means and techniques for reduction of an image to a recognizable form without loss of image specificity. The careful study of the challenge revealed the opportunity to solve it via a theory establishing reasonable ties between an image nature, IAE applications, pattern recognition philosophy, image representations and models, IAE transforms, and corresponding information technologies.

The purpose of the workshop is to discuss a methodology, mathematical and computational techniques for automation of image mining on the base of mathematical theory for IAE.

Another important task of the workshop is to discuss linguistic tools for image mining - image knowledge bases and image science ontologies - and to estimate the prospects of the algebraic approach in representation of image analysis knowledge in this environment.

The interpretation of mathematical and linguistic techniques will be illustrated by application problems.

The workshop is organized in the framework of the 3rd International Joint Conference on Computer Vision, Imaging and Computer Graphics Theory and Applications (VISIGRAPP 2010) in cooperation with the Technical Committee no. 16 "Algebraic and Discrete Mathematical Techniques in Pattern Recognition and Image Analysis" of the International Association for Pattern Recognition and with the National Committee for Pattern Recognition and Image Analysis of the Russian Academy of Sciences.

The workshop scientific program includes 3 invited talks, 12 regular papers, informal discussions, and a wrap-up session. The workshop starts with an opening session to introduce the workshop topics, goals, participants, and expected outcomes. The invited talks give overviews of the key topics. Contributed talks (selected based on review by at least two members of the program committee) represents a mix of new results based on completed work, work in progress, research challenges, and applications.

In a whole, given the increasing interest on this subject, the IMTA-workshop is intended to offer the possibility to discuss the most recent advances in this very important and challenging branch of theoretical and applied computer science.

This workshop is intended to cover, but it is not limited to, the following topics:

1. New Mathematical Techniques in Image Mining

- Algebraic Approaches

- Discrete Mathematics Techniques

- Structural and Syntactic Techniques

- Multiple Classifiers

- Other Mathematical Techniques

2. Image Models and Image Features

3. Automation of Image Mining

- Image Mining, Computer Vision and Knowledge-Based Systems

- Image Databases

- Image Knowledge Bases

- Image Mining Technologies

- Linguistic Tools

- Image Science Ontologies

- Image Science Thesauri

\section{Applied Problems}

The IMTA-Workshop Chairs:

Dr.-Eng. Igor Gurevich, Dorodnicyn Computing Center, Russian Academy of Sciences, Moscow, the Russian Federation, Professor Dr. Heinrich Niemann, Friedrich-Alexander-University of ErlangenNürnberg, Germany, Professor Ovidio Salvetti, Institute of Information Science and Technologies, Italian national Research Council. 